\title{
SPREADing the Web
}

\author{
Paul Couderc and Michel Banâtre
}

IRISA/INRIA

Campus Universitaire de Beaulieu 35042 Rennes Cedex, France

$\{$ Paul.Couderc, Michel.Banatre\}@irisa.fr

http://www.irisa.fr/aces

\begin{abstract}
This paper demonstrates the use of a physical/spatial computing model (SPREAD) to build contextual web (or wap) information systems. The approach presented consists in spreading information items in the environment (through short distance wireless technologies); then, sensing these data allows to integrate a context description, which can be used to help users co-navigate seamlessly the web as they move. The paper contributes an unified definition of the concept of context, applicable to both real (physical) and virtual (information system) environments. The underlying SPREAD system is also presented, as well as its potential for dynamic web content driven by physical entities.
\end{abstract}

\section{Introduction}

Today, ubiquitous access to information system is a reality. Systems like WAP effectively allow access to Web or Web-like information systems almost anywhere on a mobile phone or a PDA. However, we think these systems lack a crucial feature to qualify as truly pervasive information systems : context-awareness and spontaneous operation.

Context-awareness is not new, and has been extensively discussed in the academic domain. The basic motivation supporting this concept is that the most precious resource in mobile computing is user-attention: the user is focusing on his "real-life" activity, and interactions with the device are a disturbance which should be limited to the minimum. The idea behind context-awareness is to sense information from the environment of the user to guess its situation. The concept of context-awareness was born in the pioneer project Parctab at the beginning of the 1990's [1311], and many similar works followed [2]. However, context-awareness does not seem to emerge beyond the research domain.

It has been already argued [10,2] that it is the lack of models, architectures and tools which prevents context-awareness to be enabled to its full potential. The design of context-aware applications remains too difficult. The notion of context usually considered is often quite fuzzy and difficult to deal with from an information system point of view.

In this paper, we propose a different approach based on the concept of spatial information systems [5], offering several advantages. We also propose a uni- 
fied definition of context which is convenient to both physical and virtual environments. This notion of context allows to interpret mobility from a new and exciting perspective. Moreover, the concept of spatial information systems is supported by a well-understood programming model sharing similarities with LINDA [7]. This model is implemented in SPREAD (Spatial PRogramming Environment for Ambient computing Design), which allows extremely easy application developments for the spatial aspects of the context.

The paper is organized as follows : section 1 proposes to reconsider the notion of context, from the perspective of both real and virtual environments. Section 2 describes how the notion of context in the web can be enhanced with new aspects, like a spatial dimension. The SPREAD programming model is described in section 3. Section 4 concludes the paper by highlighting the innovation of our approach, and by giving some research perspectives.

\section{Context-Awareness Revisited}

The importance of context-awareness has been identified since the beginning of ubiquitous computing. The Parctab project exhibited context-sensitive applications as early as 1994 [11. However, this notion of context is still not clearly defined and understood. Some important questions, which can be easily answered in the particular case of a given application, lacks general answers and require a deeper understanding:

What does the context represent, and for who (end user, application, system ...)? What kind of information constitutes "context", how does the context relate to the notion of mobility, how does the context should be represented and used from the application perspective?

\subsection{What is "Context"?}

Context in the Real World. The first works focused on demonstrating the interest of exploiting context-awareness at the application level, enabling what is now called "pervasive computing". The considered notion of context was related to the situation of a human user in the physical environment. Basically, the idea is to guess the situation of the user in order to allow the application to operate more seamlessly. Many works followed Parctab on this principle, like the shopping companion "Shopnavi" [9] which helps a user to track shops, prices, and products using positioning and visual identification techniques, or the tourism applications CyberGuide [1] and GUIDE [3] which enhance a city visit with contextual information and navigational help.

For these applications, "context" can be defined as any information characterizing the situation of the user in the physical environment. This may include time, location, nearby physical entities (people, objects, machines, animals...)

All these information contribute to help understanding a situation from an application perspective : for example, a navigation application deals with location information, and thus the current location of the user is part of the context 
to which this application should be aware of. In this way, the context is defined "from the bottom" by a set of information (context attributes) which constitutes it. A problem in this way of defining the context is its incompleteness and lack of generality: the definition refers to the notion of "situation", which is subjective and dependent on the considered environment and applications. The definition does not help to answer some of the fundamental questions we presented earlier. In particular, it does not address the fundamental relationships between context and a mobile entity, and how an information system should integrate the notion of context.

We insist on the importance of this problem because ubiquitous computing involves a growing integration between information systems and physical environment : it may be seen as a dissolution of the information system in the real world. This trend requires to carefully examine the notion of context not only in the physical environment ("situation" of the user) but also for computing processes associated with real-world entities which evolve in the information system.

Context in Information Systems. Notions of context also exist in information systems. For example, the context of a computing process is a point in its execution graph. Another example is a link to a web page, which represents a citation context. A set of words can also be interpreted as a context, which can be found in relevant documents from a collection. It is this notion of context which is used by search engines. A last example is the context of an e-mail, which is a rich set of information: the date when it was received, the sender identity, the thread (or subject) it belongs to, etc.

As we can see, the notion of context in information system refers to the concept the location of an information item or document, relatively to a set of other information items. Thus, the context can be seen as a set of references to an information item, defining a location in an information space.

An important aspect to examine about the notion of context is its relationship in respect to the mobility. In the real world moving physically effectively changes the current context. A similar notion of mobility exists in information systems, when a process is addressing data. For example, a web browser is an agent which allows a human users to "move" inside the web space by jumping from document to document along web links, being each time exposed to a new context. This important notion of mobility inside information system was discussed by Dix and al. in [6].

Because ambient computing tends to blend the frontier between the real (physical) and virtual worlds, we think that a unified notion of context which could be applied to both kind of environments is desirable.

\subsection{Unified Context}

From the previous descriptions, we think that the notion of context refers to two main concepts: the first one is the idea of a "location" of an entity in a given 
space. The considered spaces may be of any kind: the physical space, the time, the space of idea, the space of web pages, the space of words from a language etc.

The second aspect which appears essential to us is the notion of "relativity": the notion of context involves relationships with other elements to define the location. The context cannot be considered as an "absolute location" (like a location defined by coordinates), but a relative location charaterized by the proximity of other elements.

Thus, we can define the context as the location of a (mobile) entity inside the spaces it belongs to, relatively to others objects inside these spaces.

We will not detail Spaces, which depend on the considered environment. We just need to think of them as mathematical spaces, populated with objects where relationships between these objects depend on the specific kind of spaces considered. For example, a computer memory can be seen as a one-dimensional address-space, populated with machine-word objects.

The notion of relative position means that objects of the space are comparable in some way (according to some dimension). Consider for example the physical space: one object can be compared to surrounding objects according to the euclidian distance.

More precisely, the context of an entity $e$ is defined by a set of information items

$C=\left\{x_{1}, x_{2}, \ldots, x_{n}\right\}$ such as $\forall i, d\left(e, x_{i}\right) \leq T_{i}$

Where $d$ is a distance function, giving a metric of the relative proximity of $e$ against $x_{i}$, and $T_{i}$ are thresholds defining the limits of the context.

The parameter $T_{i}$ could be used to dynamically reduce or expand the limit of the context, and thus the set of objects considered as relevant. In the case of the physical space, this parameters is supposed to be set by the user as the notion of distance is meaningful for him.

The interesting aspect of this definition is that an entity may belong to several spaces (or one space with multiple dimensions), which is especially important in the ubiquitous computing model, where information systems define information spaces related to the physical space. For example, a PDA or a cell-phone includes both attributes related to the physical environment (like the current date/time and location), and attributes related to an information system (for example, the URI of a Web page associated with the current location).

Distance functions may be defined to represent either the distance according to one dimension, or over several dimensions. For example, one may define a distance function to sort relevant web pages accordingly to both physical distance and semantic relevance $: d\left(e, x_{i}\right)=d_{\text {sem }}\left(e, x_{i}\right) / d_{p h y}\left(e, x_{i}\right)$.

\subsection{Context in the Web}

To build context-aware Web systems, and according to the previous definition of context, we have to precise the structure of the space considered. In the case of the Web, we can identify two common dimensions: the topical dimension, and the "temporal" dimension. 


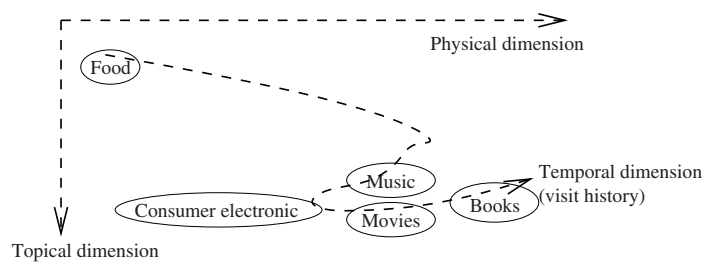

Fig. 1. Web contextual dimensions of a user visiting a shopping center

The first one refers to the topical proximity between Web pages. It may be measured by the number of hyperlink hops separating two pages, or by other means like the textual proximity between the pages (note that we could also consider two distinct dimensions, corresponding respectively to the hyperlink proximity and the textual proximity).

The temporal dimension corresponds to the information space defined by the history of the visited pages, which we all know and navigate using the "back" and "next" buttons of the Web browser.

To link the Web with the real world, we need to add a spatial dimension to the context, and associate Web pages or links with physical volumes. In such a Web system, implicit links between information are defined by the physical proximity. When navigating on such a Web system, the entity (human user, or agent) can change of navigation mode at any time, by simply switching to another dimension. Consider for example someone visiting a shopping mall enabled with such a web system; the user walks as usual to look for his favorite products, the browser navigates in spatial mode reflecting the links to nearby products. The user discovers an interesting item, but needs to have more information about the product: he just clicks the link corresponding to the product, making him "dive" into the virtual web by switching the dimension of navigation to topical. From this point, the user navigates on the Web (for example until he reaches the page of a review of the product).

The temporal dimension may be used to backtrack along both topical and physical dimensions. The figure 1 1 illustrates a web information space of a shopping center, with the pages positioned according to the different contextual dimensions (including the physical dimension). The picture shows for example that food is far away from cultural products in both physical and topical dimensions, but close in the visit history of the user: music is visited one hop after food).

\section{Building and Navigating the Web Physically}

The perspective of such a multi-dimensional Web browsing is quite exciting. Physical mobility becomes an integral part of Web browsing (the user is effectively walking on new kind of web links), while the already existing dimensions of the web enhances perception of the real world. From the perspective of our definition, the context of the mobile entity (the Web browser, which is itself an agent of the user) is its position inside a three dimensional (topical, physical 
and temporal) space. The context is thus a kind of cursor in the physical and information spaces where the mobile entity evolves.

While conceptually simple, this kind of applications will only emerge when location dependent web systems will become massively available. We propose a "physical" approach to the problem, which does not involve a logical representation of the physical space (like a Geographic information system).

\subsection{Principle}

In the physical approach, the data representing the context is implicitly specified in the physical space, by the physical arrangement of objects 54 . In such systems, control flow and information processing are directly done through physical objects placement and mobility, like in a mechanical machine, except that instead of processing physical forces it processes information.

The approach has two main advantages :

First, it is not needed to maintain the coherency between a logical representation of the physical world and the reality, which is crucial when the number of physical objects associated with information system grows, or when the objects are highly mobile.

Second, building and managing the information system is done implicitly by the management of physical objects. No work on an intermediary logical representation of the physical space is required, because the logic of the information system is represented in the physical space.

\subsection{The SPREAD Programming Model}

In our model, a spatial information system is constituted of a set of information, each information filling a sphere in the physical space. Computing in this socalled physical information system is supported by a the SPREAD middleware, which adopted a LINDA-like tuple-space approach [7].

In SPREAD, the contextual aspects of applications are programmed with a minimalist API, yet powerful. Four primitives are supported: rd and capture to read a single (respectively a set of) tuple(s), out to put a tuple in a specified physical area relatively to the publisher entity (actually a specified range, due to technological limitations), and finally drop to remove a tuple from the physical space. For more information about SPREAD, please refer to [4].

Note that when tuple range is infinite, our the primitives $r d$ and out have the same semantic as LINDA ones because the tuple space is global and independant of physical objects location.

\subsection{Spreading the Web in the Physical Space}

We used the physical information system approach to realize our contextual web system. The idea is to "spread" data defining a web context directly in the physical space. The data are called contextual clues. These contextual clues are 


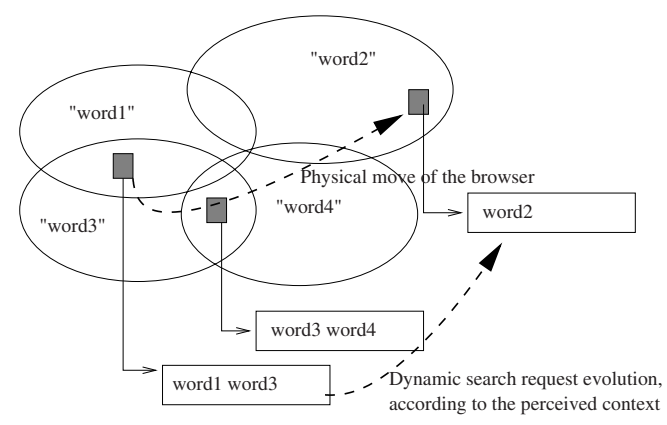

Fig. 2. Contextual analogy

published by physical objects, to cover a specified area. At a given point in the space, an application perceives a set of clues which contributes to define the context.

We used two types of clues: direct pointers, and textual clues.

Direct Pointers. In this mode, a physical object represents one or more web links (URIs). The object rays in a sphere around him (of a specified range) the URIs, which may be perceived by nearby entities (depending on the range). The resulting structure is a set of URIs filling different area in the physical space. These area may correspond to places, objects, machines, peoples and so on. As an entity moves, it navigates along the spatial dimension of the Web, implicitly existing through the surrounding objects.

Textual Clues. Direct pointers are not always adequate to describe a Web context. In many cases, a more fuzzy specification is required. For example, someone may only want to publish a specification of his interests, instead of a direct pointer to his personal Web page.

We propose the concept of contextual analogy to allow similar specification through the physical space : the proximity along the spatial dimension is considered analog to the proximity along another dimension (in this case, topical or textual dimension). Given this principle, we can fill the physical space with different clues defined by words or piece of text. A given point of the physical space will be covered by zero, one or more clues, thus defining spatially a web search request like shown on figure 2

As an entity moves, the context integrated by the application from the visible clues changes according to the surrounding objects, dynamically building the current search request. Note that is also possible to combine clues from both the physical space and a user specified request or profile to further refine spatial browsing. We experimented the concept with textual clues, but we may imagine other forms. 


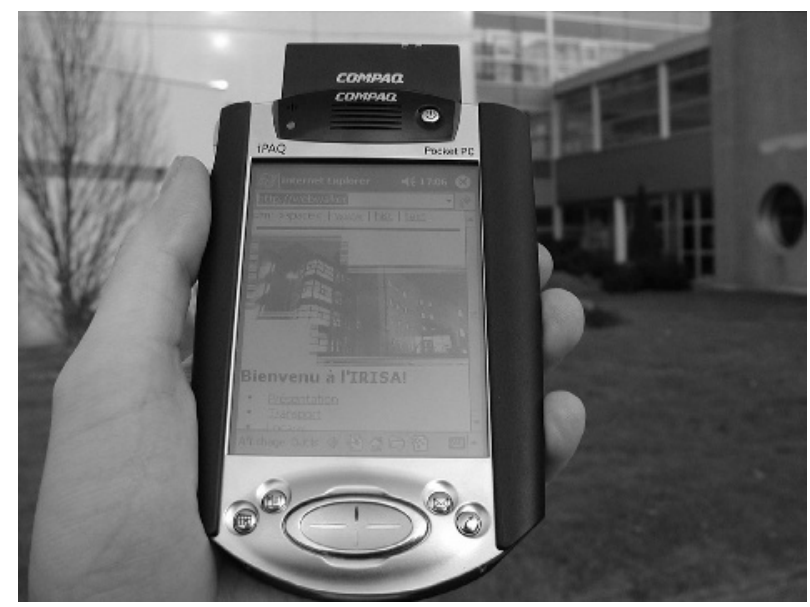

Fig. 3. Internet Explorer driven by Webwalker in spatial mode

\subsection{Programming the Web Application with SPREAD}

We will now illustrate the use of SPREAD to build the contextual web application described in the previous section.

Filling the Physical Space with Links or Clues. Spreading the existing Web into the physical space is as easy as disposing in the space physical devices enabled with our system. These devices integrate a simple application which just issues out ( $\langle t\rangle$, range) operations for each URI or clues associated with the area surrounding (which depends on the specified range) the device. The tuples are formatted as follows:

- For URI, a tuple has the form : <"URI", "http:// ..."

- For textual clues, a tuple has the form : <"TEXT_CLUE", "a piece of text">

Prototype Overview. The idea exposed previously has been implemented and experimented : the contextual web application has been developed with the SPREAD system, and experimented inside our laboratory (figure 31) and the large technological museum of La Cité des Sciences, Paris. We give here an overview of the prototype architecture.

As we said earlier, the physical objects which may participate in the spatial information system should integrate autonomous computing and communicating ability to make the physical information system possible. We use iPAQ 3600 serie PocketPC PDAs running WinCE 3.0 and IEEE 802.11B wireless LAN PCMCIA cards. 
The contextual Web browser (webwalker) is implemented as a local web proxy interfaced with Microsoft Pocket Explorer. This proxy is a SPREAD applications which integrates contextual clues from the surrounding objects. Depending on the mode of browsing, the clues (URI) are either forwarded directly to the standard web browser, or used to constitute a dynamic search request.

\section{Conclusion}

We conclude this paper by a highlighting some strong points of our approaches, and giving some research perspectives.

The idea of location sensitive Web browser was first introduced by Mobisaic [12]. The concept was refined further by the Cooltown project [8] which proposes an architecture to bridge the web to the physical world through wireless URI broadcast and sensing. The contextual Web we presented innovates in two ways :

The multi-dimensional context definition we proposed integrates the different aspects of the context, both in the existing web (virtual) and the physical world. This definition also allows to interpret mobility in this multi-dimensional space.

The second innovation resides in the physical approach we used: the information system is built from the physical space, by the contribution of many clues which are integrated to define contexts. Contextual interactions are all expressed in terms of a spatial programming model. The power of the model allows much more "spatial web applications" than browsing, like for example contextual notification in a museum or a shopping center when the user is near a bookmarked context.

From a broader perspective, the model proposed by SPREAD makes possible a radically new way of programming context-aware applications. It also allows to envisage a new concept of computing machines where information flow and actions synchronization is implicitly controlled by the placement and movements of smart mobile objects. The simplicity, power of expression and completeness of the LINDA-like programming model are strong advantages for designing and analyzing context-aware systems and spatial information systems.

An important topic of further research would be to re-consider the analysis of web accesses traces from the perspective spatial dimension. If spatial web browsing emerges, many results depending on analysis of web access locality in existing dimensions (temporal or topical) are likely to be re-considered. The spatial dimension will add complexity to these analysis as it may involve dimensions "switches", or multi-dimensional mobility : for example, a user may be browsing along the topical dimension while moving spatially.

\section{References}

1. G. D. Abowd, C. G. Atkeson, J. Hong, S. Long, R. Kooper, and M. Pinkerton. Cyberguide: A mobile context-aware tour guide. ACM Wireless Networks, 3, 1997.

2. Guanling Chen and David Kotz. A survey of context-aware mobile computing research. Technical Report TR2000-381, Dept. of Computer Science, Dartmouth College, November 2000. 
3. K. Cheverst, N. Davies, K. Mitchell, and A. Friday. Experiences of developing and deploying a context-aware tourist guide: The guide project. In MOBICOM'2000, 2000.

4. P. Couderc and M. Banâtre. Ambient computing applications: an experience with the spread approach. In Hawaii International Conference on System Sciences, January 2003.

5. Paul Couderc. Mobilité contextuelle dans les systemes d'information. $\mathrm{PhD}$ thesis, Université de Rennes-1, 2001.

6. Alan Dix, Tom Rodden, Nigel Davies, Jonathan Trevor, Adrian Friday, and Kevin Palfreyman. Exploiting space and location as a design framework for interactive mobile systems. ACM Transactions on Computer-Human Interaction, 7(3):285321, 2000.

7. David Gelernter. Generative communication in Linda. ACM Transactions on Programming Languages and Systems, 7(1):80-112, 1985.

8. T. Kindberg, J. Barton, J. Morgan, G. Becker, D. Caswell, P. Debaty, G. Gopal, M. Frid, V. Krishnan, H. Morris, J. Schettino, B. Serra, and M. Spasojevic. People, places, things: Web presence for the real world, 2000.

9. K. Nagao and J. Rekimoto. Agent augmented reality : A software agent meets the real world. In Second International Conference on Multiagent Sytems, December 1996.

10. J. Pascoe. Adding generic contextual capabilities to wearable computers. In The Second International Symposium on Wearable Computers, pages 92-99, October 1998.

11. B. N. Schilit, N. Adams, and R. Want. Context-aware computing applications. In Proceedings of the Workshop on Mobile Computing Systems and Applications, Santa Cruz, CA, December 1994.

12. G. M. Voelker and B. N. Bershad. Mobisaic - an information system for a mobile wireless computing environment. Technical Report TR-95-04-01, 1995.

13. M. Weiser. Some Computer Science Issues in Ubiquitous Computing. Communication of the ACM, 36(7):75-83, July 1993. 